\title{
Effects of stimulus-numerosity upon distance estimates
}

JOHN C. BAIRD ${ }^{1}$

WALTER REED ARMY INSTITUTE OF RESEARCH

A psychophysical approach was used to evaluate the results of reducing the number of stimuli in the visual field upon distance estimates. It was expected that decreases in numerosity would be accompanied by decreases in the magnitude of distance estimates. Numerosity reduction did have a slight but significant effect in the expected direction.

When a person moves through his environment toward a stationary point in a frontal plane, the subtended images of objects within the plane leave his visual field, and such numerosity reduction may serve as information for the judgment of physical distance traveled. Conversely, movement away from a stationary point is accompanied by an increase in stimulusnumerosity. The extension of these geometrical facts to psychophysics requires several assumptions, the most important being that conditions defining a single stimulus are constant. This excludes the possibility, for example, of a single stimulus at one distance being treated as multiple stimuli (more detail) at a closer distance.

Gibson, Olum, \& Rosenblatt (1955); Hochberg \& Smith (1955) have discussed some general consequences of stimuli leaving the visual field for distance perception, but they did not separate clearly the roles of stimulus-size and -numerosity. It is in fact technically difficult to experiment with the number of stimuli in the visual field independent of their sizes and interfigure distances (Baird, 1965) though I have been partly successful in evaluating the effects of numerosity reduction through the use of a magnitude estimation method (Stevens, 1956).

Method

The study was conducted in a dimly illuminated, $12 \times 10-\mathrm{ft}$. room, at one end of which was a homasote board (4 $x \quad 3$ ft.) standing upright on a table. A 2-in. square was removed from the center of the board and a reduction tube (20 in. long) inserted into the opening. The O's line of sight (right eye) was oriented by an adjustable headrest so he could view the stimuli, which appeared on a lighted screen (6-1/2 $\times$ 5-1/4 in.) located $4 \mathrm{ft}$. from the cornea. The screen was frosted glass presenting a constant luminance of $82.6 \mathrm{ft}$.- L as measured with a Macbeth illuminometer.

The stimuli were static, consisting of multiple outline figures drawn in black India ink (1 mm thick) upon sheets of white tracing paper. These were mounted in separate cardboard frames and were presented singly in front of the lighted screen. Four stimulus patterns were employed; a standard, and three comparisons. The standard contained 60 equilateral triangles, each $4 \mathrm{~mm}$ on a side, located within a $4 \times 4$ in. area with an equal number of figures in the top and bottom halves of the pattern. When positioned for viewing, each triangle had one side parallel to the floor. The mean inter-figure distances between centers was approximately $6.5 \mathrm{~mm}$, and the luminance of the pattern was $52.4 \mathrm{ft}$. $-\mathrm{L}$ when placed before the screen. The three comparison stimuli contained different numbers of equilateral triangles (9-mm sides). In constructing these patterns no attempt was made to exactly replicate the relative positions of the standard figures. One comparison contained 60 triangles (high numerosity) with a mean inter-figure distance of $14 \mathrm{~mm}$, and with the figures equally distributed in the top and bottom halves of the pattern. The second comparison was a subset of the first duplicated from its center and consisted of 24 triangles (medium numerosity); the third comparison was reproduced from the centers of the other two and contained six triangles (low numerosity). A piece of cardboard was available to cover the end of the reduction tube, thereby occluding $O$ 's view of the stimuli. Twelve undergraduate men served as Os. No visual tests were administered but each stated he had normal (corrected if necessary) vision.

The plan was to have Os estimate the distances of the comparisons in respect to the standard, but somewhat unusual instructions were used to match the unusualness of the task and stimulus patterns. The $O$ was told to pretend he was in a capsule moving through space and that he must estimate the distance he had traveled toward a group of planetary objects by noting changes in the figure pattern appearing on the screen before him. A magnitude estimate of distance was required (Stevens, 1956). First, he saw the standard for $10 \mathrm{sec}$. and was informed that these objects were 1000 miles away. The occluder then was placed over the end of the reduction tube and the pattern was changed to one of the comparisons, subsequently exposed for $6 \mathrm{sec}$. The $O$ verbally estimated the absolute distance of this second pattern (assumed to represent the sameobjects) relative to the standard, and following this, the remaining two comparisons were successively estimated. This entire procedure was done twice. Each $O$ was run on two of the six possible presentation orders which were randomly assigned so that every order was used four times.

As partial control, after distance estimates were completed, the four patterns were shown individually and the number of triangles in each were estimated. 
The stimuli were only visible for $4 \mathrm{sec}$, and $O$ was informed prior to stimulus exposure that he might not have time to count the triangles but he should try to do as well as he could under the circumstances. Each $O$ received one of the 24 possible presentation orders, the particular order being randomly assigned with the added constraint that an order not be repeated.

\section{Results}

If Os were responding in exact accordance with relative-size changes between the standard and comparison triangles, all distance estimates would have been 444.44 miles; whereas, the numerosity variable should alter this value so that the greater the number of triangles, the greater the distance estimate. The means obtained for the first estimates of the comparison stimuli were: low numerosity, 450; medium, 492; and high, 515; with standard deviations of 297.2, 205.1, and 193.9, respectively. For the second estimates the means were: low, 404.2; medium, 427.5; and high, 525.0 with standard deviations of $188.5,125.7$, and 108 .

Because of obvious individual differences in the range of numbers given for judgments, nonparametric statistics were applied. Ranks were assigned independently to each O's mean estimate (based on two values) of each comparison, and a Friedman Test (Siegel, 1956) was conducted to determine differences between the three numerosity levels. The sum of ranks for the low, medium and high patterns were: $20.5,19.0$, and 32.5, respectively and the obtained $\mathrm{x}_{\mathrm{r}}^{2}$ was 9.12 which with 2 df is significant $(p<.05)$. Therefore, although the combined means of the group (reported above) maintain the hypothesized relations among numerosity levels, and the Friedman Test was significant, separate ranking of $O$ 's estimates indicated no certain distinction between the distance ordering of the low and medium patterns, though the high pattern was decidely located farther away than the other two.

The mean estimates of numerosity were: standard, 45.0; high, 42.7; medium, 21.3; and low, 5.9. All Os recognized the relative numerosity differences among the three comparisons, and hence, the data were not subjected to statistical treatment.

\section{Discussion}

The hypothesis that the greater the reduction of stimulus-numerosity in a visual field, the shorter the estimated distance to those stimuli, is only partially supported by these findings. The distinction between distance rankings of low and medium numerosities was negligible, but both were usually judged closer than the high pattern.

The change in figure sizes accompanying transfer from the standard to comparisons was probably a critical factor here. Questioning of Os revealed this variation to be the most important stimulus for distance estimates. All the combined means are fairly close to the values predicted on the basis of relativesize changes, and this is not surprising in view of recent work on this cue (e.g., Baird, 1965). Despite such influences, however, the significant Friedman Test and the relative ordering of the means suggest a linkage of distance estimates with numerosity level, and the quantitative form of this function should be pursued in more detail.

\section{References}

Baird, J. C. Area and distance estimation of single and multiple stimuli. Vis. Res., 1965, 5, 661-678.

Gibson, J. J., Olum, P., \& Rosenblatt, F. Parallax and perspective during aircraft landings. Amer. J. Psychol., 1955, 68, 372-385. Hochberg, J., \& Smith, O. Landing strip marking, and the "expansion pattern": I. program, preliminary analysis, and apparatus. Percept. mot. Skills, 1955, 5, 81-92.

Siegel, S. Non-parametric statistics. New York: McGraw-Hill, 1956. Stevens, $\mathbf{S}$. S. The direct estimation of sensory magnitudes: Loudness. Amer. J. Psychol., 1956, 69, 1-25.

\section{Note}

1. I am grateful to Drs. Joseph Notterman, Frank Geldard and william Biersdorf for their comments on this research, which was conducted at Princeton University and prepared for publication at Walter Reed. My current address is: Psychological Laboratory, University of Stockholm. 